\title{
Estudos de QSAR-3D em derivados 5-nitro-2-tiofilidênicos com atividade frente a Staphylococcus aureus multi-resistente
}

\author{
Andrea Masunari e Leoberto Costa Tavares \\ Laboratório de Planejamento e Desenvolvimento de Fármacos, Departamento de Tecnologia Bioquímico- \\ Farmacêutica, Faculdade de Ciências Farmacêuticas, Universidade de São Paulo
}

*Correspondência:

L. C. Tavares

Departamento de Tecnologia

Bioquímico-Farmacêutica

Faculdade de Ciências Farmacêuticas

Universidade de São Paulo

Av. Prof. Lineu Prestes n. 580

05505-900 - São Paulo - SP, Brasil

E-mail: leoberto@usp.br
Campos moleculares extraídos de aplicativos utilizados em estudos de QSAR-3D apresentam, em geral, grande número de informações, muitas vezes irrelevantes na expressão da atividade biológica. O programa Volsurf converte as informações presentes em mapas de energia de interação tridimensionais em número reduzido de descritores bidimensionais que se caracterizam como de fácil entendimento e interpretação. Assim, foram avaliados, neste estudo, dezoito derivados 5-nitro-2-tiofilidênicos com atividade antimicrobiana frente a Staphylococcus aureus multiresistente, correlacionando as características tridimensionais destes ligantes com a referida atividade. Para o desenho e conversão tridimensional dos ligantes foram utilizados os aplicativos Sybyl (Tripos Inc) e CORINA (Molecular Networks GmbH Computerchemie), respectivamente. Os campos de interação molecular foram calculados no programa GRID (Molecular Discovery Ltd). A aplicação do programa Volsurf (Molecular Discovery Ltd) resultou em modelo estatisticamente robusto $\left(r^{2}=0,93, q^{2}=0,87\right)$ com 48 descritores estruturais, mostrando ser a hidrofobicidade propriedade fundamental no condicionamento da atividade antimicrobiana.

\section{INTRODUÇÃO}

O uso abusivo e indiscriminado de agentes antimicrobianos tem proporcionado o surgimento de resistência microbiana aos fármacos de uso corrente e, como conseqüência, determina a necessidade de busca de sucedâneos que possam vir a substituir aqueles que já não têm eficácia.

Mortes associadas a infecções bacterianas variam de $12 \%$ a $81 \%$ no mundo todo, dependendo das condições e particularidades dos tipos de pacientes e tratamentos utili- zados, observando-se que a elevação das taxas de mortalidade está associada, fundamentalmente, ao aumento do espectro de resistência dos patógenos (Diekema, Pfaller, 2003). Cerca de $50 \%$ a $87 \%$ das infecções hospitalares, têm como agente responsável o Staphylococcus aureus, sendo que em $16 \%$ a $43 \%$ dos casos os pacientes evoluem para o óbito em função do amplo espectro de resistência deste microrganismo (Metan, Zarakolu, Unal, 2005; Brenwald, Fraise, 2003; Fuchs et al., 2002; Masunari et al. 2002; Suller, Oliveira, 2001; Russel, 2000; Crowcroft, 1999). 
Na história do desenvolvimento e descoberta de fármacos antimicrobianos, inserem-se os compostos nitrofurânicos, que foram os primeiros fármacos nitroheterocíclicos introduzidos na quimioterapia e vêm sendo utilizados desde a década de 1940 para o tratamento de infecções do trato urinário (Venuti, 1995). Estudos relacionados à atividade antimicrobiana destes compostos mostraram que a maioria dos derivados ativos apresentava amplo espectro de ação, agindo não apenas sobre bactérias Grampositivas e Gram-negativas como, algumas vezes também, sobre protozoários (Gelb, 2002; Tavares et al., 1999; ElObeid, Elnima, Al-Badar, 1985; Avril et al., 1980). Compostos nitrofurânicos utilizados como antibacterianos apresentam em comum o grupo azometínico $(-\mathrm{CH}=\mathrm{N}-\mathrm{N}<)$ ligado ao carbono 2 do anel 5-nitrofurânico resultando, provavelmente, em sinergismo farmacológico entre estas duas subestruturas (Foye, Lemke, Willians, 1995; Korolkovas, 1988). Apresenta-se, na Figura 1, o grupo farmacofórico de compostos nitrofurânicos com atividade antibacteriana.

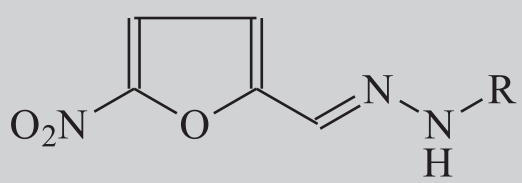

FIGURA 1 - Grupo farmacofórico de compostos nitrofurânicos com atividade antibacteriana.

Como requisito indispensável para o desencadeamento da atividade biológica desses compostos identificase a biorredução do grupo nitro, que resulta na perturbação do fluxo fisiológico de elétrons, inibindo enzimas associadas ao processo de produção de energia celular, processo vital para a manutenção bioquímica do microrganismo (Viodé et al., 1999; Tocher, 1997; Murray et al., 1996; Ulmar, Mitchard, 1968; Asnis, Gots, 1951).

Tavares e colaboradores (Tavares, Amaral, 1991; Tavares, Penna, Amaral, 1991), verificaram que a hidrofobicidade é a propriedade físico-química que mais influencia a atividade antibacteriana em série de derivados do 5-nitrofurano com estrutura análoga à nifuroxazida, 5nitro-2-furfurilideno 4-hidroxibenzidrazida (Figura 2).

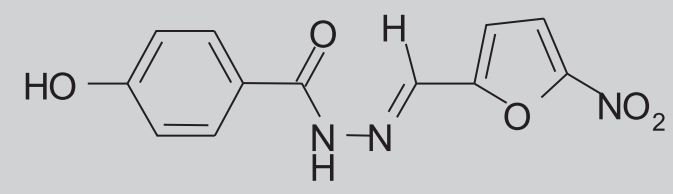

FIGURA 2 - Estrutura química da nifuroxazida
Foi verificado por esses autores que a substituição do anel furânico por tiofênico favorece significativamente a referida atividade para os compostos $p$-acetil substituídos, cujo valor de concentração inibitória mínima para o derivado tiofênico mostrou-se cerca de quatro vezes menor que o determinado para o derivado furânico. Vale ressaltar, ainda, que a 5-nitro-2-tiofilideno 4-acetilbenzidrazida se mostrou pronunciadamente mais ativa que alguns fármacos nitrofurânicos utilizados na terapêutica como a nitrofurantoína e a própria nifuroxazida (Masunari et al., 2003; Tavares et al., 1999a; Tavares, Penna, Amaral, 1997).

Comprovadas as atividades antibacteriana e antiprotozoária dos derivados 5-nitrotiofênicos estudados nesse trabalho (Masunari, 2006; Dias, 2005; Furlaneto, 2005) aliado ao fato de pesquisadores aprofundarem cada vez mais o entendimento do seu mecanismo de ação, acredita-se que estudos voltados para o aproveitamento desses compostos como fármaco constitui-se em alternativa extremamente viável para controle de doenças de caráter emergencial. Ressalta-se que no planejamento e desenvolvimento de novos agentes que sejam eficazes no tratamento de infecções causadas por bactérias multi-resistentes, observa-se alta viabilidade de aplicação de metodologias de potencialização da atividade farmacológica de compostos com perfil antimicrobiano conhecido (Fernandes et al., 1999), citando-se como vantagens deste procedimento a economia significativa de tempo e de recursos, além da grande perspectiva de sucesso (Barreiro, Fraga, 2001; Hansch, Leo, 1995).

Uma das abordagens mais utilizadas nas últimas décadas na área de desenvolvimento de novos fármacos, são os estudos de QSAR-3D (3D Quantitative StructureActivity Relationships), que passaram a apresentar maior impacto e aplicabilidade a partir da introdução do programa computacional CoMFA (Comparative Molecular Field Analysis) por Cramer e colaboradores no final da década de 80 (Cramer, De Priest, Patterson, 1993; Cramer, Patterson, Bunce, 1988). Nesta abordagem, assume-se que as interações ligante-receptor não são covalentes e que, alterações na atividade biológica podem estar relacionadas a mudanças nos campos estereoquímicos e eletrostáticos.

A evolução do conhecimento na área de biologia molecular como a caracterização precisa dos sítios de ligação em complexos ligante-receptor, contribuiu significativamente para a aplicação e entendimento da análise de QSAR-3D. Muitos métodos, entretanto, baseiam o cálculo de propriedades moleculares nas conformações absolutas dos compostos no espaço. Isso implica o fato de que a conformação selecionada para o estudo de QSAR passa a exercer papel fundamental na obtenção de dados válidos e 
torna-se, em muitos casos, fator limitante para o desenvolvimento do estudo (Martin, 1998; Kubinyi, 1997a, b).

O programa Volsurf(Cruciani et al., 2000; Cruciani, Pastor, Guba, 2000) é um método computacional que produz e explora as propriedades físico-químicas espaciais de molécula ou de um conjunto delas, a partir de mapas tridimensionais de energia de interação gerados entre moléculas e probes. O conceito básico deste programa é converter a informação presente em mapas tridimensionais gerados pelo programa GRID (Bobbyer et al., 1989; Goodford, 1985) em número reduzido de descritores de fácil interpretação. Assim, tamanho, forma, ligações de hidrogênio e hidrofobicidade podem ser quantitativamente diferenciados em uma série análoga de moléculas. Vale ressaltar que a extração de informações químicas a partir de mapas de campos moleculares e a subseqüente geração de descritores estruturais apresentam papel fundamental no processo de otimização de estruturas líderes (Cruciani et al., 2000). Além disso, modelos gerados pelo programa Volsurf são pouco influenciados pela conformação espacial dos compostos e não requerem o alinhamento das moléculas em estudo, duas vantagens que, em conjunto, viabilizam consideravelmente a utilização deste aplicativo quando não se conhece a estrutura do sítio receptor (Cruciani et al., 2000).

Com a finalidade de se determinar quantitativamente a influência de descritores estruturais de caráter físicoquímico sobre a atividade antimicrobiana de 5-nitro-2tiofilideno benzidrazidas substituídas, considerando suas características tridimensionais, desenvolveu-se o estudo de QSAR-3D por aplicação do programa Volsurf, correlacionando a atividade antimicrobiana de dezoito compostos com diferentes descritores dessas moléculas. O modelo final, com 48 descritores estruturais, apresentou-se estatisticamente robusto e com alta capacidade de predição da atividade antimicrobiana da série estudada.

A partir dos resultados obtidos neste trabalho, pode-se aprofundar, ainda mais, o estudo de características estruturais que determinam a atividade antimicrobiana de derivados 5-nitro-2-tiofilidênicos, com vistas à seleção de possíveis candidatos a análise farmacológica mais ampla.

\section{MATERIAL E MÉTODOS}

\section{Compostos estudados}

Foram estudadas dezoito 5-nitro-2-tiofilideno benzidrazidas $p$-X-substituídas, em que $\mathrm{X}=\mathrm{H}, \mathrm{Cl}, \mathrm{I}, \mathrm{Br}, n$ $\mathrm{C}_{3} \mathrm{H}_{7}, i-\mathrm{C}_{3} \mathrm{H}_{7}, n-\mathrm{C}_{4} \mathrm{H}_{9}, \mathrm{OC}_{3} \mathrm{H}_{7}, \mathrm{OC}_{4} \mathrm{H}_{9}, \mathrm{NHC}_{4} \mathrm{H}_{9}, \mathrm{CH}=\mathrm{CH}_{2}$, $\mathrm{COCH}_{3}, \mathrm{C}_{2} \mathrm{H}_{5}, \mathrm{CH}_{3}, \mathrm{CN}, \mathrm{NO}_{2}, \mathrm{SO}_{2} \mathrm{NH}_{2}$. Foi incluído, ainda, no estudo o derivado $m, p-\mathrm{Cl}_{2}$ dissubstituído. Todos os compostos foram previamente sintetizados e identificados pelo grupo de pesquisa do Laboratório de Planejamento e Desenvolvimento de Fármacos - FBT/FCF-USP (Masunari, Tavares, 2006b; Masunari et al., 2003; Rezende et al., 2002; Furlanetto, Santos, Tavares, 2001), utilizando-se as análises espectrométricas de $\mathrm{RMN}-{ }^{1} \mathrm{H}$, $\mathrm{RMN}-{ }^{13} \mathrm{C}$ e Infravermelho como metodologias de confirmação da estrutura química dos análogos. A faixa de fusão e a análise elementar foram utilizadas como avaliação do grau de pureza dos compostos.

\section{Ensaio microbiológico}

A avaliação da atividade antimicrobiana dos compostos da série estudada frente às cepas padrão (ATCC 25923) e multi-resistente (3SP/R33) de Staphylococcus aureus (Oliveira et al., 2001) foi feita por determinação da concentração inibitória mínima, CIM, aplicando método clássico de macrodiluição sucessiva em tubos (Collins, 1995; Tavares, 1993). Na primeira etapa, fase I, determinou-se a atividade antimicrobiana com fator de diluição de ordem 2 . Na segunda etapa, fase II, utilizou-se metodologia adaptada por Tavares e colaboradores (Tavares, 1993; Tavares, Penna, Amaral, 1997), que consiste no estreitamento da faixa de concentração determinada na fase I, com o propósito de intensificar a sensibilidade do ensaio.

\section{Estudo de QSAR-3D}

O estudo de QSAR-3D foi realizado em Estação de Trabalho Power Challenge 100 da Silicon Graphics, 4 CPU 194 MHz MIPS 10000, 512 MB de memória RAM e sistema operacional IRIX 6.5. Esta etapa do estudo foi realizada no Laboratório de QSAR/QSAR 3-D no Departamento de Química Fundamental do Instituto de Química da Universidade de São Paulo. Utilizou-se o programa Volsurf (Cruciani et al., 2000, Cruciani, Pastor, Guba, 2000), cuja metodologia está fundamentada na conversão de informações presentes em mapas de campos moleculares tridimensionais em um número reduzido de descritores bidimensionais. Ressalta-se que o Volsurf não possui o módulo de conversão de estruturas bidimensionais para estruturas tridimensionais, tornando necessária, desta forma, a utilização de aplicativos desenvolvidos para esta finalidade como CONCORD ou CORINA, para a posterior análise de imagem.

As estruturas químicas dos dezoito análogos em estudo foram construídas, inicialmente, no programa Sybyl versão 6.9.1. (Tripos Inc) e importadas para o programa CORINA versão 3.0 (Molecular Networks GmbH Computerchemie) para a geração das estruturas 
tridimensionais. O CORINA se baseia em regras fixas préestabelecidas, que têm como referência dados de cristalografia de raios-X. Este programa considera aspectos conformacionais dos ligantes e os submete a configurações de energia mínima.

Determinadas as estruturas tridimensionais dos ligantes no CORINA, os arquivos foram exportados para o programa Volsurf, versão 3.0.11 (Molecular Discovery Ltd.) para início dos cálculos. O programa GRID constitui parte integrante deste e possui a função de gerar os campos de interação molecular conhecidos como MIF's (Molecular Interactions Fields). Para a geração dos MIF's utilizou-se uma caixa de pontos com espaço entre os nós de $0,5 \AA$ e foram selecionados quatro probes. A saber: $\mathrm{H}_{2} \mathrm{O}$ (probe hidrofílico), DRY (probe hidrofóbico), $\mathrm{NH}$ amídico (doador de ligações de hidrogênio) e CO carbonílico (aceptor de ligações de hidrogênio). Utilizou-se para os cálculos valores de atividade biológica na forma de potência antimicrobiana (log 1/CIM).

O processo de validação dos modelos obtidos foi feito por análise de PLS (Partial Least Squares) (Wold, Johanson, Cocchi, 1993, Geladi, Kowalski, 1986), cuja aplicação é recomendada em casos em que se dispõe de um número de dados experimentais muito inferior ao número de descritores gerados. Este algoritmo é altamente recomendado em estudos de QSAR-3D pelo fato de que, ao contrário da regressão linear múltipla (RLM), dados com alta correlação (colinearidade) e/ou ruído ou, ainda, muitas variáveis $\mathrm{X}$ (descritores estruturais) podem ser analisadas.

Todo padrão de performance do PLS pôde ser avaliado pela análise conjunta da relevância de cada descritor estrutural gerado, pela observação do gráfico de $\mathrm{Y}_{\text {calc. }}$ vs $\mathrm{Y}_{\text {exp }}$ (potência antimicrobiana calculada pelo modelo versus potência antimicrobiana determinada experimentalmente) e pelo gráfico de SDEC (desvio padrão do erro de cálculo) $v s$ SDEP (desvio padrão do erro de predição), que indica para cada componente analisado o valor de $\mathrm{R}^{2}$ (variância explicada no ajuste) e de $\mathrm{Q}^{2}$ (variância explicada na predição). Para o estudo da magnitude da influência dos descritores gerados sobre a potência antimicrobiana da série estudada foi analisado, ainda, o gráfico de coeficientes do modelo de PLS.

\section{RESULTADOS E DISCUSSÃO}

\section{Síntese e avaliação da atividade antimicrobiana}

Foram sintetizadas e testadas, neste estudo, quatorze 5-nitro-2-tiofilideno benzidrazidas, sintetizadas em três etapas, a saber: obtenção de benzoatos de metila (2) a partir de ácidos benzóicos, obtenção de benzidrazidas (3) a par- tir de benzoatos de metila e obtenção de bases de Schiff(4) a partir de benzidrazidas substituídas e 5-nitro-2tiofenocarboxaldeído (Tavares et al., 1999; Masunari et al., 2003) (Figura 3).

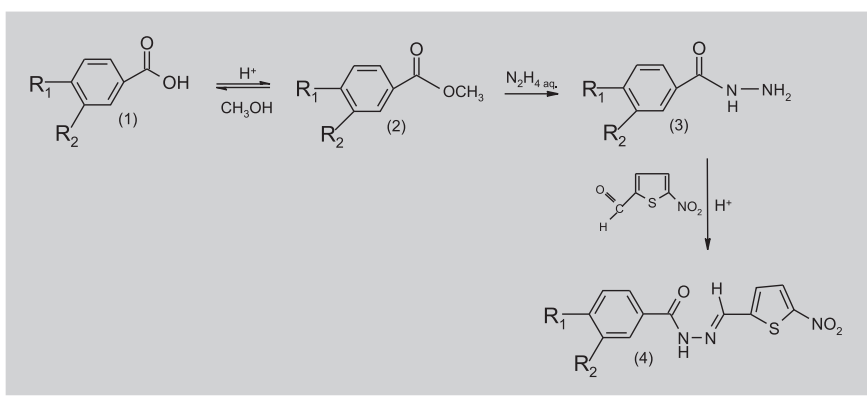

FIGURA 3 - Rota de síntese para a obtenção das 5-nitro2-tiofilidenobenzidrazidas.

A síntese se mostrou simples, prática e com rendimentos satisfatórios, apresentando valores na faixa entre $80 \%$ e $90 \%$ para a maioria dos compostos obtidos (Masunari et al., 2003).

Para a avaliação da atividade antimicrobiana, determinou-se a concentração inibitória mínima, CIM, frente às cepas padrão (ATCC 25923) e multi-resistente (3SP/R33) (Oliveira et al., 2001) de Staphylococcus aureus seguindo metodologia de macrodiluição sucessiva adaptada por Tavares e colaboradores (Tavares, Penna, Amaral, 1997).

Entre os compostos testados frente ambas as cepas de Staphylococcus aureus constatou-se que a 5-nitro-2tiofilideno-4-acetilbenzidrazida (XIV) é o composto mais ativo enquanto que a 5-nitro-2-tiofilideno-4butilbenzidrazida (XI) é o composto de mais baixa atividade (Tabela I).

Os resultados obtidos para a cepa multi-resistente (Tabela 1), sugerem que o derivado não substituído (I) apresenta primordialmente atividade bacteriostática, já que o valor de CBM determinado é muito maior que o de CIM. Por outro lado, os derivados $p$ - $\mathrm{Br}(\mathrm{V}), p-\mathrm{Cl}$ (II), $m, p-\mathrm{Cl}_{2}$ (III) e $p-\mathrm{COCH}_{3}$ (XIV) exibiram excelente atividade bacteriostática e exerceram efeito bactericida em concentrações relativamente baixas, mostrando que estes derivados são mais potentes entre os compostos estudados.

\section{Estudos de QSAR-3D}

Após a conversão dos dezoito derivados 5-nitro-2tiofilidênicos em estudo em estruturas tridimensionais utilizando o programa CORINA (Sadowski, Rudolph, Gasteiger, 1992; Gasteiger, Rudolph, Sadowski, 1990), foram gerados os mapas de campos de interação molecular por aplicação do programa GRID (Bobbyer et al., 1989; 
TABELA I - Concentração inibitória mínima, CIM, de 5-nitro-2-tiofilideno benzidrazidas frente às cepas ATCC 25923 e $3 \mathrm{SP} / \mathrm{R} 33^{\mathrm{I}}$ de Staphylococcus aureus ${ }^{\mathrm{II}}$

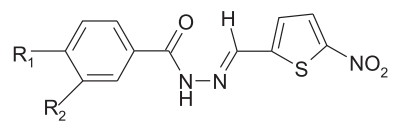

\begin{tabular}{|c|c|c|c|c|c|}
\hline \multirow[t]{2}{*}{$\mathrm{N}^{0}$} & \multirow[t]{2}{*}{$\mathrm{R}_{1}$} & \multirow[t]{2}{*}{$\mathrm{R}_{2}$} & Cepa ATCC 25923 & \multicolumn{2}{|c|}{ Cepa 3SP/R33 } \\
\hline & & & $\operatorname{CIM}(\mu \mathrm{g} / \mathrm{mL})^{*}$ & $\mathrm{CIM}(\mu \mathrm{g} / \mathrm{mL})^{*}$ & $\mathrm{CBM}(\mu \mathrm{g} / \mathrm{mL})^{* *}$ \\
\hline I & $\mathrm{H}$ & $\mathrm{H}$ & $\leq 4,59$ & $\leq 7,81$ & $\geq 125,00$ \\
\hline II & $\mathrm{Cl}$ & $\mathrm{H}$ & $\leq 1,71$ & $\leq 2,18$ & $4,37-2,18$ \\
\hline III & $\mathrm{Cl}$ & $\mathrm{Cl}$ & $\leq 0,76$ & $\leq 0,63$ & $0,63-1,27$ \\
\hline IV & $\mathrm{I}$ & $\mathrm{H}$ & $\leq 5,62$ & $\leq 11,10$ & $>88,80 * * * *$ \\
\hline V & $\mathrm{Br}$ & $\mathrm{H}$ & $\leq 0,75$ & $\leq 1,25$ & $1,25-0,63$ \\
\hline VI & $n-\mathrm{C}_{3} \mathrm{H}_{7}$ & $\mathrm{H}$ & $\leq 11,60$ & $\leq 14,50$ & $>29,00 * * * *$ \\
\hline VII & $i-\mathrm{C}_{3}^{3} \mathrm{H}_{7}^{\prime}$ & $\mathrm{H}$ & $\leq 9,06$ & $\leq 10,04$ & $>40,16 * * * *$ \\
\hline VIII & $\mathrm{OC}_{3} \mathrm{H}_{7}$ & $\mathrm{H}$ & $\leq 8,10$ & - & —*** \\
\hline IX & $\mathrm{OC}_{4}^{3} \mathrm{H}_{9}^{\prime}$ & $\mathrm{H}$ & $\leq 12,30$ & $\leq 20,62$ & $>41,24 * * * *$ \\
\hline $\mathrm{X}$ & $\mathrm{NHC}_{4} \mathrm{H}_{9}$ & $\mathrm{H}$ & $\leq 7,75$ & $\leq 12,45$ & $>49,80 * * * *$ \\
\hline XI & $n-\mathrm{C}_{4} \mathrm{H}_{9}$ & $\mathrm{H}$ & $\leq 13,50$ & $\leq 25,40$ & $>50,80 * * * *$ \\
\hline XII & $\mathrm{C}_{2} \mathrm{H}_{5}$ & $\mathrm{H}$ & $\leq 6,75$ & $\leq 11,60$ & $>92,80 * * * *$ \\
\hline XIII & $\mathrm{CH}=\mathrm{CH}_{2}$ & $\mathrm{H}$ & $\leq 4,72$ & $\leq 6,75$ & $>54,00 * * * *$ \\
\hline XIV & $\mathrm{COCH}_{3}^{2}$ & $\mathrm{H}$ & $\leq 0,14$ & $\leq 0,22$ & $0,44-0,22$ \\
\hline & nitrofurantoína & & 16,00 III & - & \\
\hline & nifuroxazida & & $3,60^{\mathrm{IV}}$ & - & \\
\hline
\end{tabular}

Notas: * Leituras de inibição/crescimento realizadas após 18 horas de incubação a $35^{\circ} \mathrm{C} ; * *$ As leituras da concentração bactericida mínima, $\mathrm{CBM}$, foram realizadas após 24 horas de incubação a $35^{\circ} \mathrm{C}$; *** $\mathrm{CIM}$ não determinada devido à insolubilidade do derivado nas concentrações requeridas no ensaio; **** compostos que não apresentaram atividade

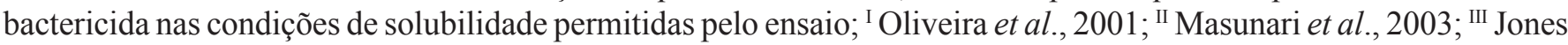
et al., 1993; ' ${ }^{\mathrm{V}}$ Tavares, Penna, Amaral, 1997.

Goodford, 1985), sendo estes posteriormente convertidos, por aplicação do programa Volsurf, em 48 descritores estruturais de caráter físico-químico.

Procedeu-se a análise de PLS (van de Waterbeemd, 1996; Wold, Johanson, Cocchi, 1993; Geladi, Kowalski, 1986) para relacionar os dados experimentais de potência antimicrobiana (matriz Y) com a matriz X de descritores moleculares. Vale ressaltar que as variáveis latentes geradas no PLS nada mais são que a combinação linear das variáveis X originais, sendo o número de variáveis latentes significativas determinado por validação cruzada. Utilizouse a opção leave one out (Cruciani et al., 2000; Wold, Johanson, Cocchi, 1993; Geladi, Kowalski, 1986), que consiste na construção de modelos que prevêem o valor de atividade biológica dos compostos em estudo, neste caso potência antimicrobiana, excluindo temporariamente um dos compostos da análise. Este processo foi repetido até que cada um dos dezoito compostos estudados fosse pelo menos uma vez excluídos da construção dos modelos.
Inicialmente gerou-se modelo bruto com 88 descritores estruturais e duas componentes principais (CP's). Apesar do valor de $\mathrm{R}^{2}=0,91$ apresentar-se aceitável, indicando boa correlação entre os valores de potência determinados experimentalmente e os valores calculados pelo modelo, observou-se valor de $\mathrm{Q}^{2}=0,26$, muito baixo, explicitando, assim, sua baixa capacidade de predição.

Observando o gráfico de $\mathrm{Y}_{\text {calc }}$. vs $\mathrm{Y}_{\text {exp }}$. para duas CP's (Figura 4), evidenciou-se o $p$-acetil derivado como um composto com comportamento fora da linha de tendência dos demais. Ressalta-se que o valor de SDEP, desvio padrão do erro de predição, equivalente a 0,40 , mostrou-se também muito elevado, corroborando o péssimo grau de predição do modelo bruto.

A classificação do derivado $p$-acetil substituído (XIV) como um provável outlier no estudo em três dimensões corrobora o estudo clássico anteriormente realizado por nosso grupo (Masunari, Rezende, Tavares, 2004; Masunari, Tavares, 2006a), em que se constatou, para este 


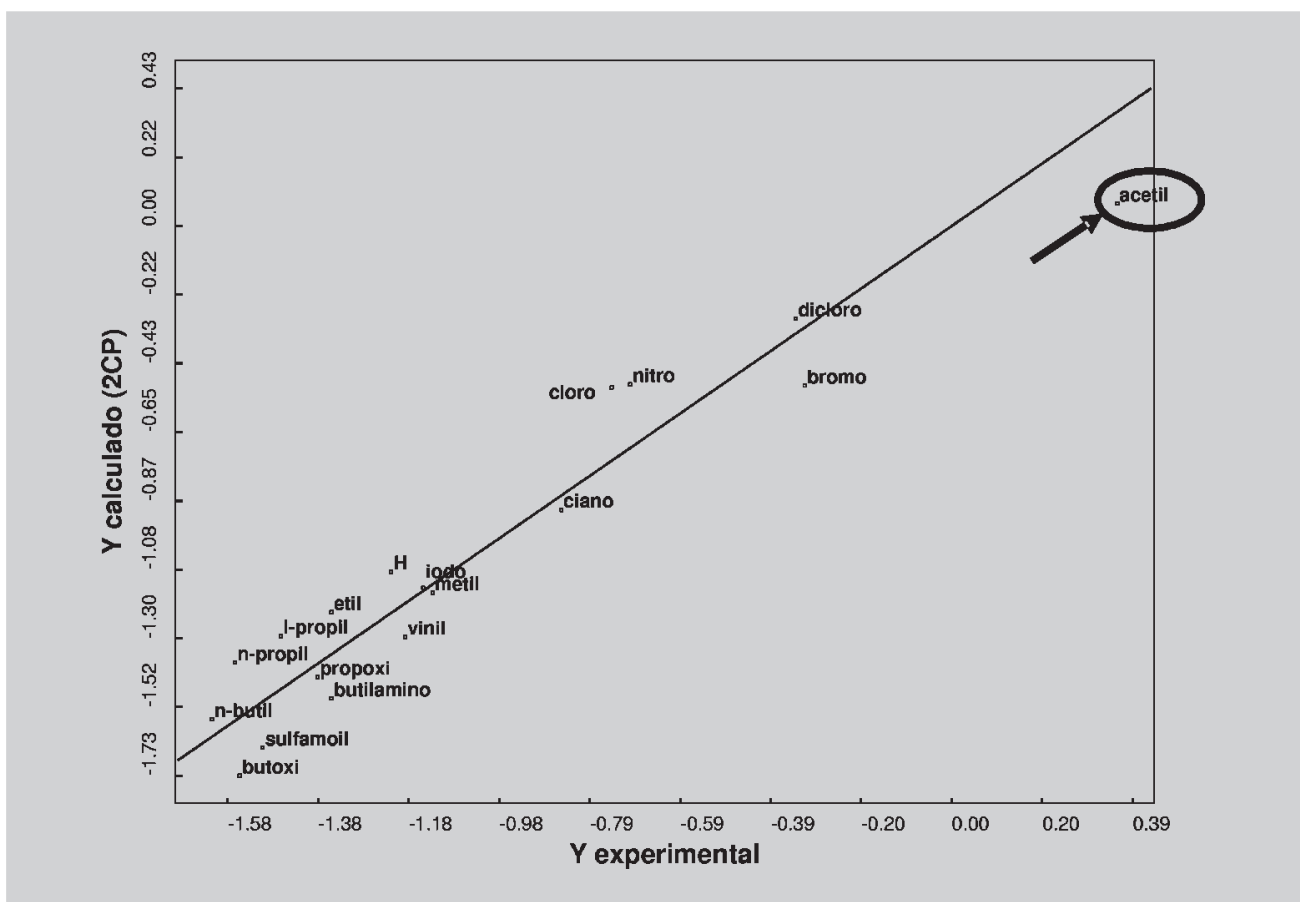

FIGURA 4 - Valores de potência antimicrobiana calculados $\left(\mathrm{Y}_{\text {calc }}\right)$ pelo modelo bruto com 2 CP's versus potência antimicrobiana determinada experimentalmente.

análogo, padrão de comportamento totalmente diferenciado dos demais componentes da série.

Retirando o $p$-acetil derivado (XIV) do modelo, observou-se melhora significativa dos parâmetros estatísticos, ressaltando, em especial, os valores de $\mathrm{Q}^{2} \mathrm{e}$ de SDEP, que passaram de 0,26 e 0,40 para 0,80 e 0,17 , respectivamente.

A atividade antimicrobiana do $p$-acetil derivado (XIV) se mostrou flagrantemente acima da linha de tendência dos demais componentes da série, permitindo, com isso, classificá-lo como super ativo. Com base em estudos realizados até momento, no entanto, não se pode afirmar, com segurança, quais seriam as interações envolvidas ou quais seriam as principais propriedades físico-químicas responsáveis por tão pronunciada atividade. Acredita-se que o efeito de campo do grupo acetil, somado a um balanço lipofílicohidrofílico adequado deste composto provavelmente estariam contribuindo para tão pronunciada atividade. Ressaltase também que para que o $p$-acetil derivado seja classificado como um outlier, ainda são necessárias informações mais aprofundadas referentes ao comportamento deste composto.

Notou-se como limitação do segundo modelo gerado, entretanto, o número elevado de componentes principais envolvidas, já que os melhores parâmetros estatísticos foram obtidos para $6 \mathrm{CP}$ 's. Ressalta-se que um grande número de CP's torna a análise mais difícil, já que quanto mais se adicionam componentes principais para explicar um modelo, maior será o risco de resultar em ajuste forçado do modelo aos dados. Obviamente, como conseqüência direta do ajuste forçado, observa-se decréscimo da validade do modelo final.

A dificuldade citada provavelmente se relaciona ao fato de a probabilidade de alguns dos descritores gerados no modelo bruto causarem sobreposição de informações, tornando evidente, em alguns casos, a redundância de dados. De fato, de acordo com Cruciani e colaboradores (Cruciani et al., 2000; Cruciani, Pastor, Guba, 2000), em uma análise de PLS é muito comum que se obtenha na matriz de descritores (matriz X) um número considerável de variáveis que não apresentam, necessariamente, relação direta com a atividade biológica, proporcionando, desta forma, uma descrição estrutural inconsistente para aplicação em estudos de QSAR-3D. Sabe-se, ainda, que é praticamente impossível a geração de bons modelos de PLS quando uma única variável significativa possa ser detectada entre outras, ainda que esta seja altamente relacionada com a atividade.

Para simplificar o modelo gerado pelo PLS, sem prejudicar seu grau de predição e significância, utilizam-se procedimentos matemáticos de seleção de variáveis contidos neste mesmo programa. No caso deste estudo utilizouse o FFD (Fractional Fatorial Design) no qual são selecionadas apenas as variáveis que aumentam a capacidade preditiva do modelo de PLS. Aplicou-se o FFD na opção fold over design, que consiste na repetição de todas as combinações de variáveis invertendo o padrão de sinais 
na matriz de combinação de forma a resultar em avaliação muito mais segura do efeito de uma variável ou de um conjunto delas na capacidade de predição do modelo. A aplicação do FFD resultou em redução considerável do número de variáveis, de 88 para 48, eliminando informações redundantes presentes no modelo bruto.

Classificou-se como melhor modelo que correlacionou a potência antimicrobiana com a estrutura tridimensional da série de compostos estudada, o modelo com 48 descritores estruturais e com valores de $\mathrm{R}^{2}=0,93$ $\mathrm{e}^{2}=0,87$ para três CP's, sendo importante citar que o alto valor do coeficiente de correlação de predição ao quadrado $\left(\mathrm{Q}^{2}\right)$ demonstra a excelente capacidade de predição do modelo. Ressalta-se, ainda, que o baixo valor de SDEP (desvio padrão do erro de predição), em torno de 0,14 , corrobora a capacidade preditiva do modelo final. Apresentase, na Figura 5, o gráfico dos valores de potência antimicrobiana experimentais e calculados pelo modelo final obtido no Volsurf para três CP's.

A aplicação do FFD possibilitou a simplificação do modelo bruto inicialmente obtido sem, entretanto, comprometer seu significado e capacidade de predição. De acordo com o gráfico dos coeficientes de PLS (Figura 6), observou-se a contribuição positiva dos descritores CW1 (fator de capacidade), D1-D8 (distribuição de regiões hidrofóbicas), CP (Critical Packing), HB2 (doador de ligações de hidrogênio) e W2 (distribuição de regiões hidrofílicas) sobre a bioatividade dos compostos representada pela potência antimicrobiana. Observou-se, por outro lado, contribuição negativa dos descritores W5-W8 (distribuição de regiões hidrofílicas), IW1-IW3 (Integy-Interaction Energy -Moments Hidrofílicos), CW2-CW7 (fator de capacidade); ID1-ID8 (Integy - Interaction Energy Moments Hidrofóbicos), HB4-HB8 (doador de ligação de hidrogênio), W3-W8 (distribuição de regiões hidrofílicas), HB7 (aceptor de ligações de hidrogênio).

Descritores gerados pelo Volsurf apresentam significados claros que se referem tanto à distribuição de regiões hidrofóbicas e hidrofílicas quanto em relação à formação de ligações de hidrogênio. Os descritores W se referem ao tamanho das regiões hidrofílicas distribuídas pelos ligantes, enquanto os descritores D se referem ao tamanho das regiões hidrofóbicas. O parâmetro de critical packing, $\mathrm{CP}$, se refere à razão entre porções $\mathrm{D}$ e $\mathrm{W}$ dos ligantes e os Integy moments hidrofílicos (IW) e hidrofóbicos (ID) medem o desequilíbrio entre o centro de massa dos compostos e o baricentro das regiões hidrofílicas e hidrofóbicas, respectivamente (Cruciani et al., 2000; Cruciani, Pastor, Guba, 2000).

O parâmetro de critical packing, $\mathrm{CP}$, em conjunto com o parâmetro de tamanho das regiões hidrofóbicas, D, são excelentes para prever a capacidade de partição em

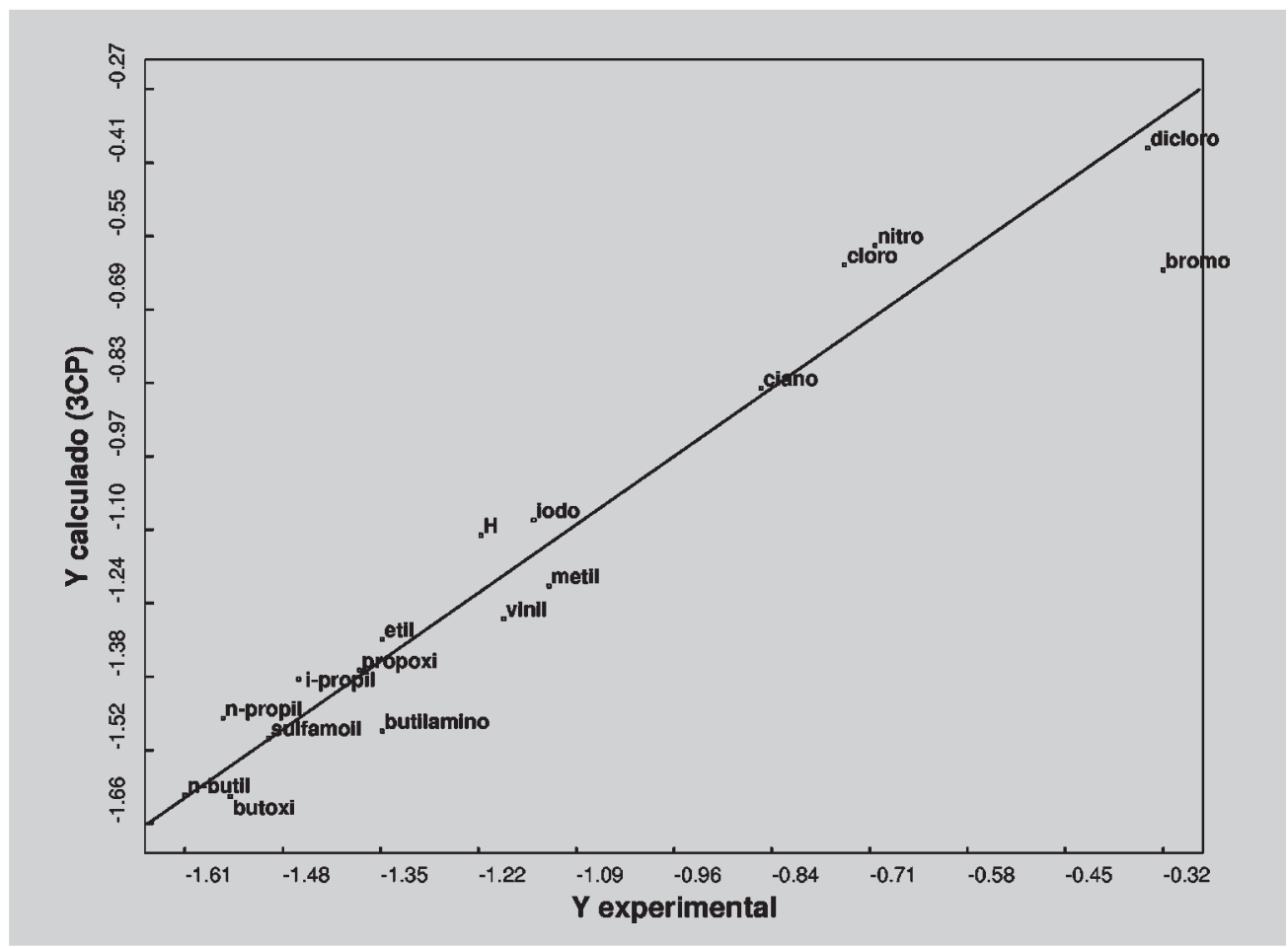

FIGURA 5 - Valores de potência antimicrobiana calculados $\left(\mathrm{Y}_{\text {calc }}\right)$ por modelo gerado no Volsurf com 3CP's versus potência antimicrobiana determinada experimentalmente $\left(\mathrm{Y}_{\mathrm{exp}}\right)$. 


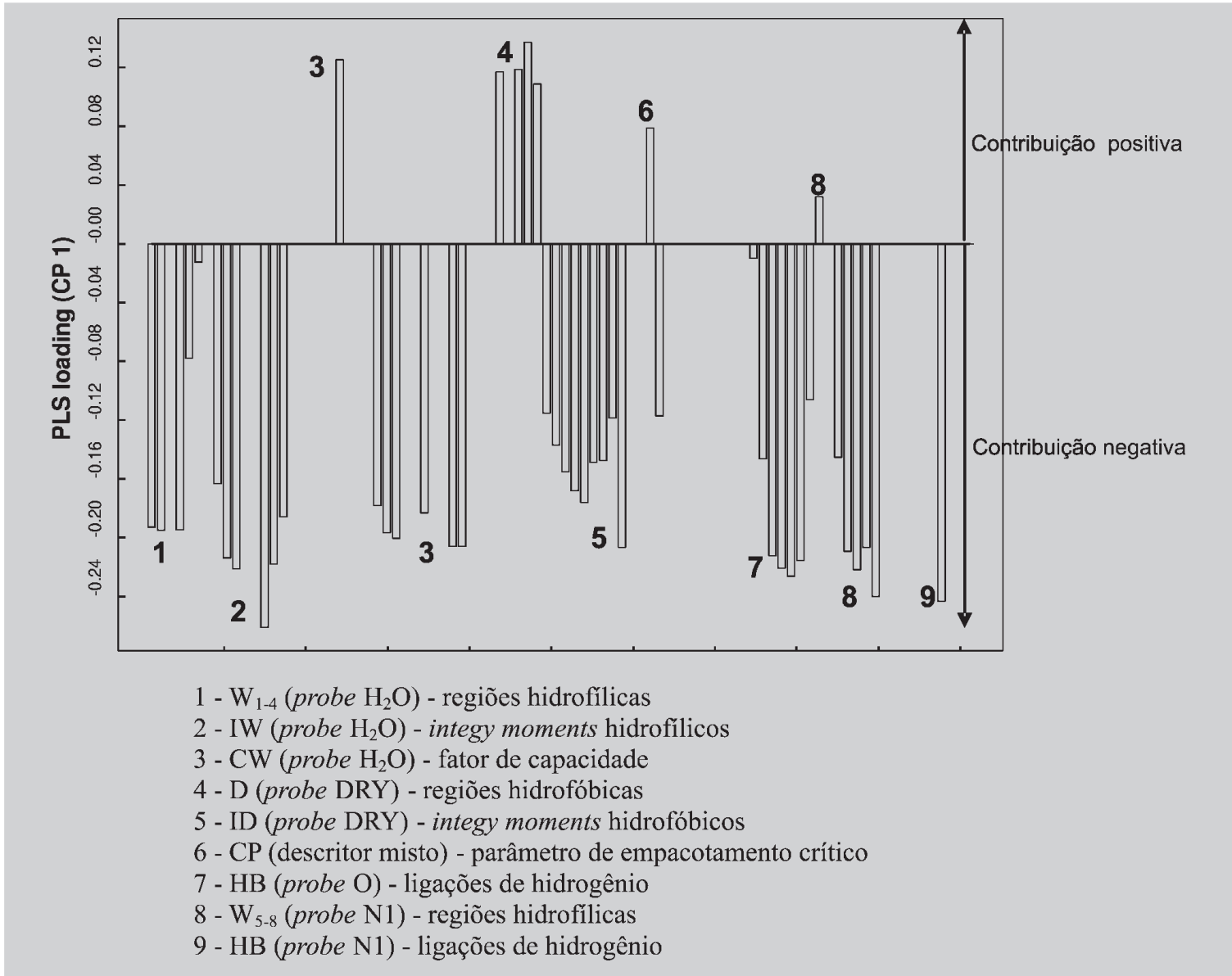

FIGURA 6 - Gráfico dos coeficientes de PLS para os descritores gerados no Volsurf.

membranas, cita-se que o CP é um descritor adimensional, que descreve a interação entre um composto anfifílico e uma membrana biológica. Esta interação depende da área hidrofilica molecular, do volume da porção hidrocarbônica e o máximo de comprimento que a cadeia pode assumir (Cruciani et al., 2000; Cruciani, Pastor, Guba, 2000).

Os integy moments se assemelham aos momentos dipolares, altos valores de IW indicam alta concentração de regiões hidrofílicas em determinadas porções das moléculas, enquanto que valores elevados de ID indicam a clara concentração de porções hidrofóbicas nos ligantes (Cruciani et al., 2000; Cruciani, Pastor, Guba, 2000).

Os fatores de capacidade, $\mathrm{CW}$, representam a razão entre regiões hidrofílicas e a superfície dos compostos em estudo. Em outras palavras é determinada a quantidade de regiões hidrofílicas por unidade de superfície, sendo a mesma proporcional à concentração de grupos polares expostos em relação a toda área molecular superficial (Cruciani et al., 2000; Cruciani, Pastor, Guba, 2000).

Os descritores HB estão diretamente relacionados com a quantidade de átomos doadores e/ou aceptores de ligações de hidrogênio nos ligantes. Os descritores de regiões hidrofílicas incluem um envelope molecular acessível a moléculas de água, ressaltando ainda o fato de que estes são, em sua maioria, relevantes na determinação da partição em membranas, nas quais processos de solvatação e dessolvatação são críticos (Cruciani et al., 2000; Cruciani, Pastor, Guba, 2000)

A contribuição negativa intensa dos descritores IW (2) e ID (5) indicam que a presença de concentração tanto de regiões hidrofílicas como de regiões hidrofóbicas nos compostos estudados resulta em decréscimo significativo da potência antimicrobiana. A contribuição positiva do descritor $\mathrm{D}$, por outro lado, que se refere à distribuição de regiões hidrofóbicas nos ligantes, indica que se uniformemente distribuída, esta propriedade tende a aumentar a potência dos compostos.

De fato, observa-se, na Figura 7, que compostos com alto valor de IW, denotados por vetores que apontam do centro de massa do ligante em direção à concentração de regiões hidrofílicas, apresentaram menores valores de potência antimicrobiana, enquanto que derivados com distri- 

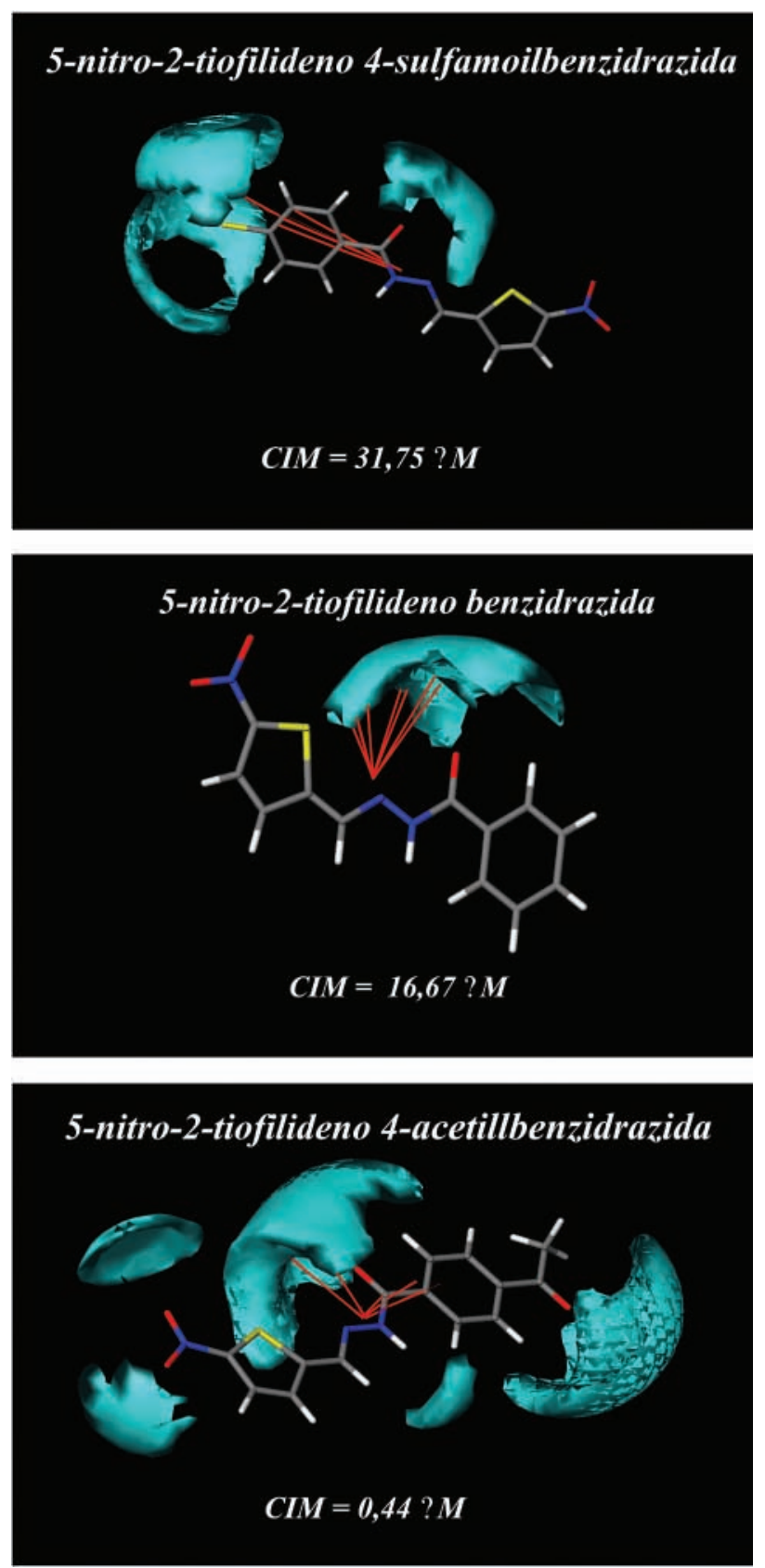

FIGURA 7 - Representação esquemática dos integy moments hidrofílicos (IW), representados por vetores em vermelho, dos derivados $p$-sulfamoil, $p$-acetil substituídos e do composto não substituído.

buição uniforme da hidrofobicidade e baixa concentração de regiões hidrofílicas resultaram em valores maiores de atividade biológica (Figura 8).

Observa-se, pela Figura 8, que a alta concentração de regiões hidrofílicas proporcionada pelo substituinte diretamente ligado ao anel benzênico da 5-nitro-2-tiofilideno 4sulfamoilbenzidrazida contribui consideravelmente para o decréscimo de potência constatado para este composto.
A contribuição positiva de CW1 e W2 provavelmente se relaciona à pequena, mas absolutamente necessária, quantidade de regiões hidrofílicas por unidade de superfície presentes nas moléculas, sendo estas essenciais para a difusão dos compostos através das membranas biológicas mediadas por processos de solvatação e dessolvatação.

A contribuição positiva do parâmetro de critical packing, CP, que nada mais é do que a razão entre porções hidrofóbicas e hidrofílicas dos ligantes, indica, em conjunto com o descritor $\mathrm{D}$, a importância da distribuição uniforme da hidrofobicidade nos ligantes bem como da presença de alguns pontos hidrofílicos nos derivados para que ocorra o processo de difusão através das membranas biológicas.

Vale ressaltar que os dados de atividade biológica utilizados neste trabalho se referem à potência antimicrobiana das 5-nitro-2-tiofilideno benzidrazidas substituídas. Este tipo de medida da atividade é amplo não podendo ser atribuído exclusivamente a processos farmacocinéticos ou farmacodinâmicos. De fato, este tipo de medida engloba provavelmente ambos processos, permitindo-nos atribuir a relevância dos descritores gerados no Volsurf à capacidade de difusão dos compostos nas membranas biológicas para posterior desencadeamento da atividade farmacológica.

Como citado anteriormente, os descritores HB estão relacionados a subunidades dos compostos em que existe a possibilidade de formação de ligações de hidrogênio. Observou-se a contribuição positiva dos descritores HB2 (probe O) e HB7 (probe N), ressaltando a capacidade doadora e receptora de hidrogênio dos compostos da série em estudo.

Os descritores relacionados ao caráter doador e receptor de ligações de hidrogênio gerados pelo programa Volsurf apresentaram influência diferenciada sobre a potência antimicrobiana. No caso do descritor HB2, que se refere ao caráter doador de ligação de hidrogênio, observou-se contribuição positiva para a atividade, enquanto que no caso do descritor $\mathrm{HB} 7$, relacionado ao caráter receptor de ligação de hidrogênio, evidenciou-se contribuição negativa intensa em relação à atividade.

A partir destes resultados pode-se inferir duas informações distintas e de extrema relevância: a primeira delas estaria relacionada à importância da disponibilidade do hidrogênio ligado ao nitrogênio azometínico (A) para formação de ligações de hidrogênio, enquanto que a contribuição negativa intensa do descritor HB7 sugere que ligações de hidrogênio envolvendo os grupos carbonila (B) e nitro (C) certamente resultaria em queda pronunciada da atividade biológica (Figura 9).

A partir do estudo de QSAR-3D realizado neste trabalho, por aplicação do programa Volsurf, obtiveram-se algumas informações estruturais da série de compostos estu- 

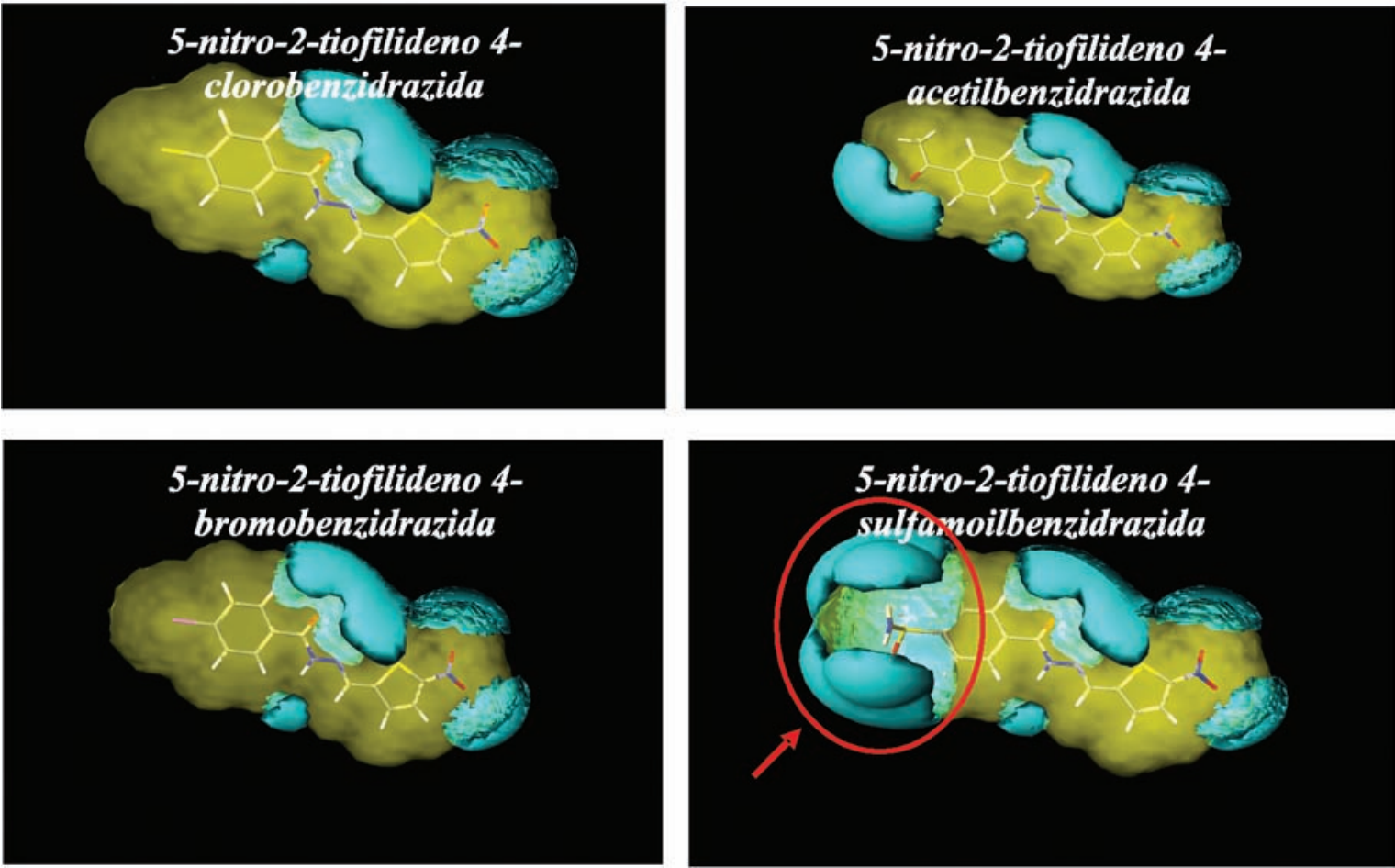

FIGURA 8 - Representação esquemática da distribuição da hidrofobicidade (amarelo) e da hidrofilicidade (azul) nas 5nitro-2-tiofilideno benzidrazidas substituídas.
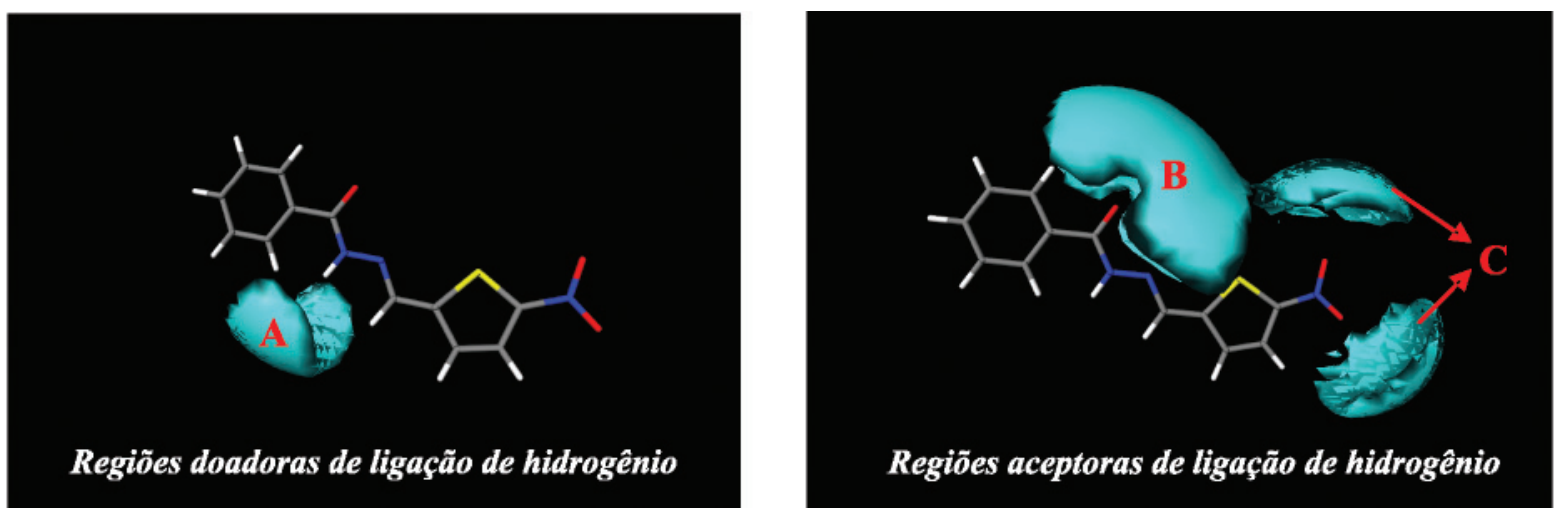

FIGURA 9 - Regiões com capacidades doadora (A) e aceptora (B, C) de ligações de hidrogênio das 5-nitro-2-tiofilideno benzidrazidas substituídas.

dada que são fundamentais para o entendimento do seu mecanismo de interação com a biofase. Em concordância com o estudo clássico (QSAR-2D), anteriormente por nós realizado (Masunari, Rezende, Tavares, 2004; Masunari, Tavares, 2006a) evidenciou-se a hidrofobicidade como propriedade fundamental na determinação de bons perfis de atividade antimicrobiana dos compostos estudados. A grande vantagem da presente abordagem em relação ao estudo de QSAR-2D, entretanto, está relacionada ao maior detalhamento da influência da hidrofobicidade sobre a referida atividade. Pôde-se especificar, por meio dos descritores gerados pelo Volsurf, que a influência positiva da hidrofobicidade estaria relacionada à distribuição uniforme desta propriedade na superfície dos ligantes, já que a presença de regiões hidrofóbicas concentradas influenciam negativamente a atividade antibacteriana.

Verificou-se, ainda, que a presença pontual de regiões hidrofílicas é fator de essencial importância, já que processos de solvatação e dessolvatação exercem papel crítico na difusão dos compostos através das membranas biológicas. Com isso, pode-se afirmar que a aplicação do estudo de QSAR-3D evidenciou a notória importância de um 
balanço lipofílico-hidrofílico adequado para um bom desempenho das 5-nitro-2-tiofilideno benzidrazidas substituídas como agentes antimicrobianos

\section{CONCLUSÃO}

No estudo de QSAR-3D desenvolvido com aplicação do programa Volsurf, evidenciou-se que a hidrofobicidade se mostra como propriedade fundamental para o condicionamento da atividade antimicrobiana de 5-nitro-2-tiofilideno benzidrazidas substituídas. Esta abordagem permitiu, também, compreensão mais detalhada de como esta hidrofobicidade influencia a bioatividade dos compostos da série estudada, constatando que bons perfis de atividade antimicrobiana estão relacionados não apenas com a distribuição uniforme da hidrofobicidade na superfície dos ligantes, mas também com a presença de regiões hidrofilicas pontuais. As regiões hidrofílicas estariam diretamente associadas a processos de solvatação e dessolvatação para a difusão através de membranas biológicas, fator essencial no mecanismo de transferência do ligante até o seu sítio de ação.

\section{ABSTRACT \\ 3D QSAR studies of 5-nitrothiophene derivatives with antimicrobial activity against multidrug- resistant Staphylococcus aureus}

Studies in three-dimensional molecular fields generally contain a large amount of data, some of which are redundant or not relevant. The program Volsurf, a quite fast method, is able to compress the relevant information present in $3 D$ molecular structures into a few easy bidimensional descriptors. This study correlates the antimicrobial activity of eighteen 5-nitro-2-thiophylidene derivatives against multidrug-resistant Staphylococcus aureus with threedimensional molecular fields of these ligands. For molecular structures sketching and 3D conversion, Sybyl and CORINA programs were used, respectively. The GRID force field was applied to generate the $3 D$ interaction energies. The Volsurf characterization results on significant statistic model with 48 descriptors $\left(r^{2}=0,93, q^{2}=0,87\right)$, observing a significant influence of hydrophobic properties on antimicrobial activity performance.

UNITERMS: 3D QSAR. Volsurf Program. Staphylococcus aureus/resistance. Nifuroxazide.

\section{AGRADECIMENTOS}

Os autores agradecem à CAPES e à FAPESP pelo suporte financeiro para o desenvolvimento deste trabalho e, em especial, à Profa. Dra. Antônia Tavares do Amaral, coordenadora do Laboratório de QSAR/QSAR - 3D do Departamento de Química Fundamental do Instituto de Química/USP, pela permissão de uso da infra-estrutura necessária para a realização dos estudos computacionais. Agradecem, também, a Leandro de Rezende e ao mestre Alberto Malvezzi, pela indispensável colaboração na realização e interpretação dos dados obtidos.

\section{REFERÊNCIAS BIBLIOGRÁFICAS}

ASNIS, R.E.; GOTS, J.S. Studies on the action of nitrofurans on bacterial enzime sistems. I. The inhibition of bacterial respiration by furacin. Arch. Biochem., New York, v. 30, n. 1, p. 25-33, 1951.

AVRIL, J.L.; BRIFFORD, J.; BEINIS, J.P.; DUBRISAY, J. Etude de l'influence de l'antibiothérapie sur les résistances des entérobactéries de l'íntestin. Ann. Microbiol., Paris, v. 131B, n. 1, p. 21-29, 1980.

BARREIRO, E.J.; FRAGA, C.A.M. Química Medicinal: as bases moleculares da ação dos fármacos. Porto Alegre: Artmed Editora, 2001. 83p.

BOOBBYER, D.N.A.; GOODFORD, P.J.; MCWHINNIE, P.M. New hydrogen-bond potential for use in determining energetically favourable binding sites of molecules of known structure. J. Med. Chem., Columbus, v. 32, n. 5, p. 1083-1094, 1989.

BRENWALD, N.P.; FRAISE, A.P. Triclosan resistance in methicillin-resistant Staphylococcus aureus (MRSA). J. Hosp. Infect., Londres, v. 55, n. 2, p. 141-144, 2003.

COLLINS, C.H.; LYNE, P.M.; GRANGE, J.M. Collins and Lyne's microbiological methods. 7 ed. Oxford: Butterworth-Heinemann, 1995. p. 493.

CRAMER, R.D.; PATTERSON, D.E.; BUNCE, J.D. Comparative molecular field analysis (CoMFA). 1. Effect of shape on binding of steroids to carrier proteins. J. Am. Chem. Soc., Columbus, v. 110, n. 18, p. 5959-5967, 1988.

CRAMER, R.D.; De PRIEST, S.A; PATTERSON, D.E. The developing practice of comparative molecular field analysis. In: KUBINYI, H. (Ed.) $3 D$ QSAR in Drug Design: Theory, Methods and Applications. Leiden: ESCOM, 1993. p. 443-485. 
CROWCROFT, N.S. Methicillin-resistant $S$. aureus and antimicrobial use in Belgian hospital., Infect. Control Hosp. Epidemiol., Thorofare, v. 20, n. 1, p. 31-36, 1999.

CRUCIANI, G.; PASTOR, M.; GUBA, W. Volsurf: a new tool for the pharmacokinetic optimization of lead compounds. Eur. J. Pharm. Sci., Paris, v. 11, n. 2, p. S29S39, 2000.

CRUCIANI, G.; CRIVORI, P.; CARRUPT, P.A.; TESTA, B. Molecular fields in quantitative structure-permeation relationships: the Volsurf approach. J. Mol. Struc. THEOCHEM, Amsterdam, v. 503, n. 1-2, p. 17-30, 2000.

DIAS, M.M.L. Pró-fármacos de derivados 5-nitro-2tiofilidênicos: planejamento, síntese e determinação da atividade antichagásica dos ligantes. São Paulo, 2005. 123p. [Dissertação de Mestrado. Faculdade de Ciências Farmacêuticas. Universidade de São Paulo].

DIEKEMA, D.J.; PFALLER, M.A. Infection control epidemiology and clinical microbiology. In: MURRAY, R.P.; BARON, J.E.; JORGENSEN, J.H.; PFALLER, M.A.; TOLKEN, R.H. (Eds.). Manual of clinical microbiology. 8 ed., Washington: American Society for Microbiology, 2003. p. 129-139.

El-OBEID, H.A.; ELNIMA, E.I.;AL-BADR,A.A. Synthesis and antimicrobial activity of new furan derivatives. Pharm. Res., Stuttgart, v. 1, p. 42-43, 1985.

FERNANDES, P.B.; MENZEL, R.; HARDY, D.J.; TSEDINH, Y.; WARREN, A.; ELSEMORE, D.A. Microbial resistance: novel screens for a contemporary problem. Med. Res. Rev., New York, v. 19, n. 6, p. 559-568, 1999.

FOYE, W.O.; LEMKE, T.L.; WILLIANS, D.A. Principles of medicinal chemistry. 4.ed. Baltimore: Williams \& Wilkins, 1995. 995p.

FUCHS, P.C.; KOOP, J.; HAFNER, H.; KLEINER, U.; PALLUA, N. MRSA - retrospective analysis of an outbreak in the burn centre Aachen. Burns, Wiltshire, v. 28 , n. 6 , p. 575-578, 2002.

FURLANETTO, M.; SANTOS, M.G.B.; TAVARES, L.C. Avaliação da influência de grupos substituintes sobre a atividade antimicrobiana de derivados 5-nitro-2tiofilidênicos. Braz. J. Pharm. Sci., São Paulo, v. 37, supl. 1, p. 62, 2001.
FURLANETTO, M. Coacervação complexa de compostos nitro-heterocíclicos. Obtenção de microcápsulas e avaliação da atividade antichagásica. São Paulo, 2005. 99p. [Dissertação de Mestrado. Faculdade de Ciências Farmacêuticas. Universidade de São Paulo].

GASTEIGER, J.; RUDOLPH, C.; SADOWSKI, J. Automatic generation of $3 \mathrm{D}$ atomic coordinates for organic molecules. Tethraedron Comp. Method., v. 3, p. $537-547,1990$.

GELADI, P.; KOWALSKI, B.R. Partial least-squares regression: a tutorial. Anal. Chim. Acta, Amsterdam, v. 185 , p. $1-17,1986$.

GELB, M.H. Drugs to combat tropical protozoan parasites. Science, Washington, v. 297, p. 343-344, 2002.

GOODFORD, P.J. Computational procedure for determining energetically favourable binding sites on biologically important macromolecules. J. Med. Chem., Columbus, v. 28, n. 7, p. $849-857,1985$.

HANSCH, C.; LEO, A. Exploring QSAR: fundamentals and applications in chemistry and biology. Washington: American Chemical Society, 1995. 557 p.

JONES, M.E.; BOENINK, N.M.; VERHOEF, J.; KOHRER, K.; SCHIMITZ, F.J. Multiple mutations conferring ciprofloxacin resistance in Staphylococcus aureus demonstrate long-term stability in an antibiotic-free environment. J. Antimicrob. Chemother., Oxford, v. 45, n. 3, p. 353-356, 2000.

KOROLKOVAS, A. Essentials of medicinal chemistry. 2. ed. New York: John Wiley, 1988. 1204 p.

KUBINYI, H. QSAR and 3D QSAR in drug design. Part 1: methodology. $D D T$, Kidlington, v. 2, n. 12, p. 538-546, $1997 \mathrm{a}$

KUBINYI, H. QSAR and 3D QSAR in drug design. Part 2: applications and problems. DDT, Kidlington, v. 2, n. 11, p. 457-467, 1997b.

MARTIN, Y.C. 3D QSAR: Current State, Scope and Limitations. In: KUBINYI, H.; FOLKERS, G.; MARTIN, Y.C. (Eds.) 3D QSAR in Drug Design, Great Britain: Kluwer Academic Publishers, 1998, v. 12-14, p. 3-23. 
MASUNARI, A.; VESSONI-PENNA, T.C.; MAMIZUKA, E.M.; TAVARES, L.C. New 5-nitro-2-thiophylidene derivatives with activity against multi-resistant strains of Staphylococcus aureus In: INTERNATIONAL SYMPOSIUM ON STAPHYLOCOCCI AND STAPHYLOCOCCAL INFECTIONS, 10., Tsukuba, 2002. Abstracts. Tsukuba: ISSSI 112-07, 2002. p.47.

MASUNARI,A.; NASCIMENTO, F.L.L.; FURLANETTO, M.; SANTOS, M.G.B.; PENNA, T.C.V.; MAMIZUKA, E.M.; TAVARES, L.C. Determinação da atividade antimicrobiana de derivados 5-nitro-2-tiofilidênicos frente às cepas padrão e multi-resistente de Staphylococcus aureus, São Paulo. Rev. Bras. Cienc. Farm., São Paulo, v. 39, supl. 3, p. 192-194, 2003. (VII Semana de Ciência e Tecnologia da FCF/USP,7., 2003).

MASUNARI, A.; REZENDE, P.; TAVARES, L.C. QSAR studies of nifuroxazide analogs with antimicrobial activity against multidrug-resistant S. aureus In: The $15^{\text {th }}$ European Symposium on Quantitative Structure-Activity Relationships \& Molecular Modeling, 15., Istanbul, 2004. Resumos de Trabalhos. Istanbul: Prous Science, 2004. p. 123.

MASUNARI, A.; TAVARES, L.C. Aplicação de estudos de QSAR-2D em derivados 5-nitro-2-tiofilidênicos com atividade antimicrobiana frente a Staphylococcus aureus multi-resistente (MRSA). Rev. Bras. Cienc. Farm., São Paulo, v. 42, n. 2, p. 203-214, 2006a.

MASUNARI,A.; TAVARES, L.C. Síntese e determinação da atividade antimicrobiana de derivados 5-nitro-2tiofilidênicos frente a Staphylococcus aureus multiresistente. Rev. Bras. Cienc. Farm., São Paulo, v. 42, n. 3, p. 461-472, 2006b.

METAN, G.; ZARAKOLU, P.; UNAL, S. Rapid detection of antibacterial resistance in emerging Gram-positive cocci. J. Hosp. Infect., London, v. 61, p. 93-99, 2005.

MURRAY, R.J.; LIM, T.T.; PEARSON, J.C.; GRUBB, W.B.; LUM, G.D. Community-onset methicillin-resistant S. aureus bacteremia in Northern Australia. Int. J. Infect. Dis., Chicago, v. 8, n. 5, p. 275-283, 2004.

OLIVEIRA, G.A.; DELL'AQUILA,A.M.; MASIERO, R.L.; LEVY, C.E.; GOMES, M.S.; CUI, L.; HIRAMATSU, K.; MAMIZUKA, E.M. Isolation in Brazil of nosocomial Staphylococcus aureus with reduced susceptibility to vancomicin. Infect. Contr. Hosp. Epidem., Thorofare, v. 22, n. 7, p. 443-448, 2001.
REZENDE, P.; MASUNARI, A.; SANTOS, M. G. B.; MAMIZUKA, E. M.; VESSONI-PENNA, T. C.; TAVARES, L. C. Hansch Analysis of nifuroxazide analogues with antimicrobial activity against MRSA. Drugs Fut., v. 27, p. 192-192, 2002. (Symposium on Medicinal Chemistry, 17., Barcelona, 2002).

SADOWSKI, J.; RUDOPLH, C.; GASTEIGER, J. The generation of 3D models of host-guest complexes. Anal. Chim. Acta, Amsterdam, v. 265, n. 2, 1992.

SULLER, M.T.E.; RUSSEL, A.D. Triclosan and antibiotic resistance in Staphylococcus aureus. J. Antimicrob. Chemother., Oxford, v. 46, n. 1, p. 11-18, 2000.

VENUTI, M.C. The role of recombinant DNA technology in medicinal chemistry and discovery. In: WOLFF, M.E. (Ed.) Burger's medicinal chemistry and drug discovery: principles and practice. 5 ed. New York: John Wiley \& Sons, 1995. v. 1, cap. 16, p. 661-696.

TAVARES, L.C.; AMARAL, A.T. Partition coefficient determination of nitrofurans derivatives for a QSAR study. In: WORKSHOP ON CHEMICAL STRUCTURE-BIOLOGICAL ACTIVITY, São Paulo, 1991. Resumos de Trabalhos. São Paulo: I.Q./USP, 1991. p. 8.

TAVARES, L.C.; VESSONI-PENNA, T.C.; AMARAL,A.T. A QSAR study of nitrofurans derivatives with antimicrobial activity. In: WORKSHOP ON CHEMICAL STRUCTURE-BIOLOGICAL ACTIVITY, São Paulo, 1991. Resumos de Trabalhos. São Paulo: I.Q./USP, 1991. p. 2.

TAVARES, L.C. Relações quantitativas entre a estrutura química e a atividade antimicrobiana de análogos a nifuroxazida. São Paulo, 1993. 186p [Tese de Doutorado. Faculdade de Ciências Farmacêuticas. Universidade de São Paulo].

TAVARES, L.C.; PENNA, T.C.V.; AMARAL, A.T. Synthesis and biological activity of nifuroxazide and analogs. Boll. Chim. Farm., Milano, v. 136, n. 3, p. 244249, 1997.

TAVARES, L.C.; CHISTÉ, J.J.; SANTOS, M.G.B.; PENNA, T.C.V. Synthesis and biological activity of nifuroxazide and analogs II. Boll. Chim. Farm., Milano, v. 138, n. 8, p. 432-436, 1999. 
TOCHER, J.H. Reductive activation of nitroheterocyclic compounds. Gen. Pharmacol., Oxford, v. 28, n. 4, p. 485487, 1997.

VIODÉ, C.; BETTACHE, N.; CENAS, N.; KRAUTHSIEGEL, R.L.; CHAVIÉRE, G.; BALAKARA, N.; PÉRIE, J. Enzymatic reduction studies of nitroheterocycles. Biochem. Pharmacol., Oxford, v. 57, n. 5, p. 549-557, 1999.

ULMAR, T.M.; MITCHARD, M. The competitive inhibition of nitroredutase by some analogues of nitrofurantoin. Biochem. Pharmacol., New York, v. 17, p. 2057-2060, 1968.
VAN DE WATERBEEMD, H. Chemometric Methods used in Drug Design. In: VAN DE WATERBEEMD, H. (Ed.) Structure-property correlations in drug research. San Diego: Academic Press, Inc., 1996. p. 55-80.

WOLD, S.; JOHANSON, E.; COCCHI, M.; PLS-Partial least squares projections to latent structures. In: KUBINYI, H., (Ed.). 3D QSAR in drug design: theory, methods and applications. Leiden: ESCOM, 1993.p. 523563.

Recebido para publicação em 13 de setembro de 2006. Aceito para publicação em 12 de dezemrbro de 2006. 